\title{
ANÁLISE FÍSICA E MATEMÁTICA DA TURBULÊNCIA NA INTERFACE METAL/ESCÓRIA NA REGIÃO DO MENISCO EM MOLDE DE LINGOTAMENTO CONTÍNUO*
}

\author{
José Dimas de Arruda ${ }^{1}$ \\ Alexandre Leopoldo Pereira ${ }^{2}$ \\ Amanda Aparecida Fátima Arruda ${ }^{2}$ \\ Samuel da Silva de Souza ${ }^{2}$ \\ Carlos Antônio da Silva ${ }^{3}$ \\ Itavahn Alves da Silva ${ }^{3}$ \\ Varadarajan Seshadri
}

\section{Resumo}

O conhecimento do campo de fluxo dentro do molde de lingotamento contínuo pode oferecer uma oportunidade de se melhorar a limpidez interna do produto. Modelagem física, aplicação da técnica PIV juntamente com a modelagem matemática via Ansys Fluent foram empregadas para avaliar a distribuição de velocidades na região de interface metal escória num molde de lingotamento de placas. Os resultados foram analisados com base em critérios de estabilidade da interface metal - escória disponíveis na literatura e ressaltam o papel do empuxo e tensão interfacial.

Palavras-chave: Lingotamento contínuo; Molde; Escória; PIV.

\section{PHYSICAL AND MATHEMATICAL ANALYSIS OF TURBULENCE AT THE METAL/SLAG INTERFACE IN THE MENISCUS REGION OF A CONTINUOUS CASTING MOLD}

\section{Abstract}

The knowledge of the flow field inside the mold can offer an opportunity to improve operations and as a result to achieve better inclusion control. Physical modeling, PIV technique and CFD using Ansys Fluent have been employed to assess the velocity distribution in the metal slag interface region in a slab mold. Results have been analyzed using stability criteria available in the literature and highlight the effects of buoyancy and interfacial tension.

Keywords: Continuous casting; Mold; Slag; Steel; PIV.

Engenheiro Metalúrgico, Mestrando em Engenharia de Materiais, Redemat, Universidade Federal de Ouro Preto, Ouro Preto, Minas Gerais, Brasil.

Graduando em Metalurgia, Escola de Minas, Ouro Preto, Minas Gerais, Brasil.

3 Engenheiro Metalúrgico, Ph.D., Professor, Universidade Federal de Ouro Preto, Escola de Minas, Ouro Preto, Minas Gerais, Brasil.

4 Engenheiro Metalúrgico, Dr. Ing., Professo Emérito, Universidade Federal de Minas Gerais (UFMG), Belo Horizonte, Minas Gerais, Brasil. 


\section{INTRODUÇÃO}

O fluxo de aço, na cavidade do molde, é por natureza, turbulento e induz instabilidades fluidodinâmicas. Isto se traduz em vórtices, fluxos recirculatórios e tensões de cisalhamento na interface aço/escória, entranhamento de escória, formação de ganchos (hooks) e outros fenômenos, Figura 1(a).
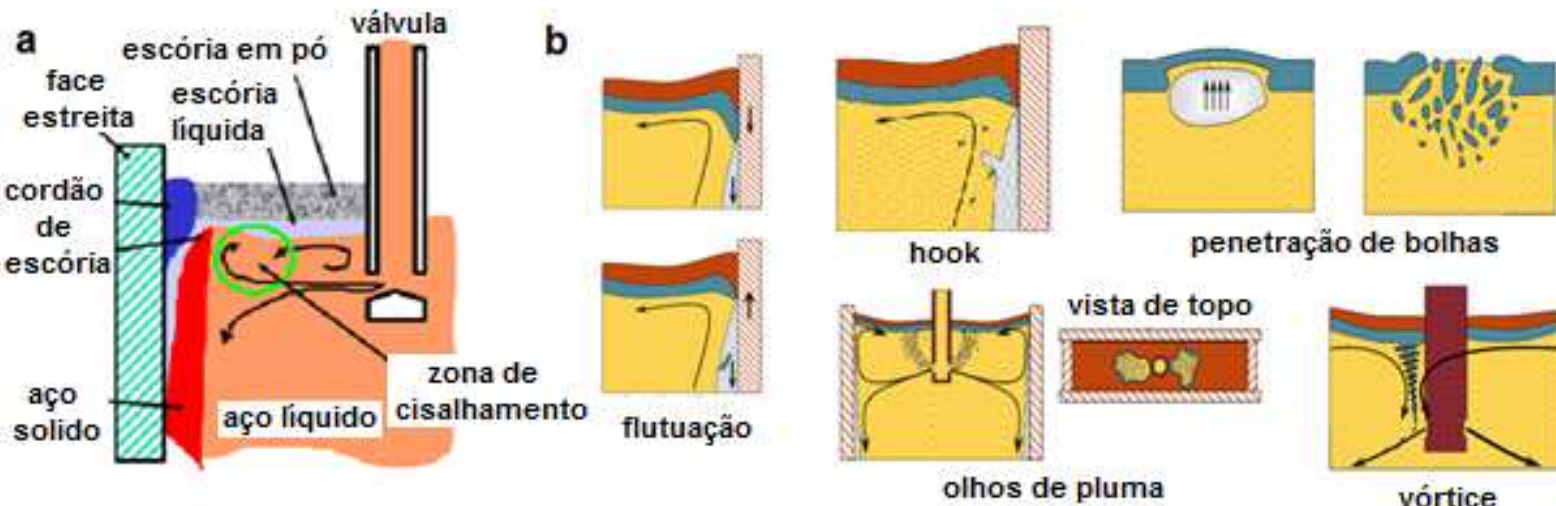

penetração de bolhas

Figura 1.a) Fenômenos ocorrentes no interior do molde de lingotamento contínuo [1]; b) Entranhamento de escória devido a mecanismos de arraste [2].

Zare [3] utilizou computação tridimensional da região de interface metal/escória, por meio do método VOF (Volume of Fluid) em conjunção com modelo k-€ de turbulência, e comparou os resultados com aqueles de um modelo físico, Figura 3a. Similarmente, Barral [4] obteve o perfil da interface água/óleo na modelagem física e matemática, identificando que, nas regiões onde a pressão obtida da modelagem matemática foi maior, a espessura da película de óleo obtida via modelagem física foi menor, demonstrando claramente o efeito do fluxo na movimentação do óleo, Figura 3b.
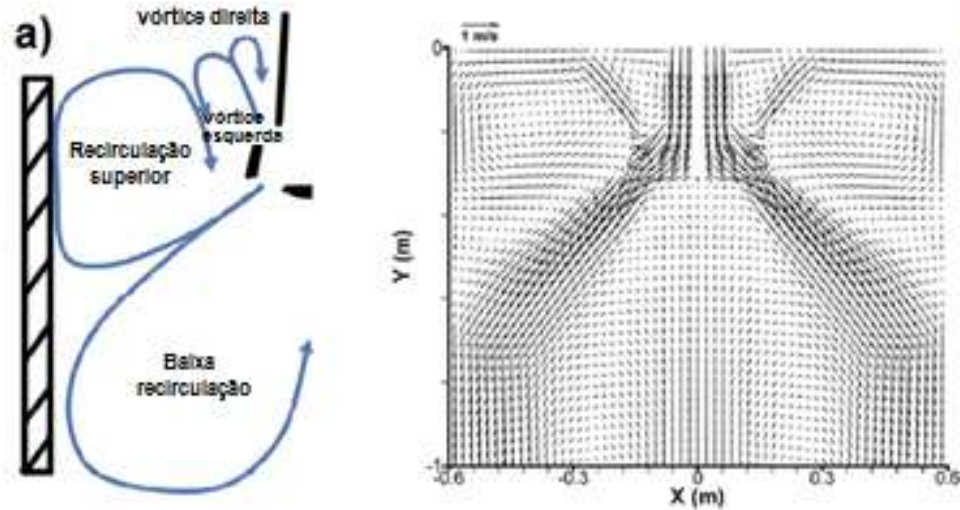

b)
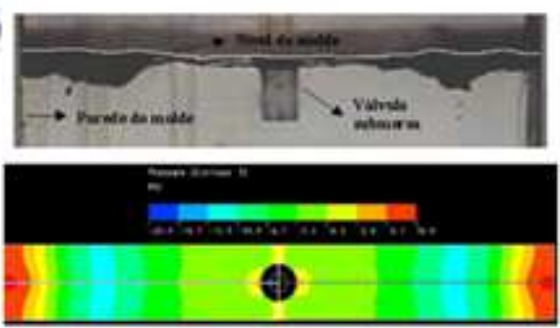

Figura 2. a) Comparação do campo de fluxo entre o modelo analítico e numérico [3]; b). Comparação do perfil de interface água e óleo na modelagem física e matemática [4].

Este trabalho emprega técnicas de modelagem física e matemática para estudar a instabilidade na interface metal - escória em um molde de placas.

\section{MATERIAIS E MÉTODOS}

Um modelo físico em acrílico, construído em escala $\lambda=0,6$, Figura 3(a) foi utilizado neste trabalho. Os adimensionais de Froude e Weber foram empregados como 
critério de similaridade. O mapa de velocidades foi determinado por meio da técnica PIV. Utilizou-se um aparelho DANTEC - 2D, o qual compõe-se de sistema laser Dual power- 65/15 400mJ, com duração de pulsos de 4ns, faixa de comprimento de onda entre $1064 \mathrm{~nm}$ e $532 \mathrm{~nm}$, além de uma câmera CCD Flow-Sense 2ME. A análise de imagens foi realizada via software Dynamics Studio - Dantec. Para todas as condições experimentais, foram adquiridas cerca de 100 imagens com intervalo de tempo de $3000 \mu$ s e freqüência de captura10Hz. Uma região quadrada de lado igual a $600 \mathrm{~mm}$, Figura 3b, sob a região do menisco, foi utilizada para monitoração do fluxo de aço entre a válvula submersa e a face estreita. Para realizar a modelagem matemática foi editado um setup utilizando um quarto do domínio de integração do molde de LC utilizado na modelagem física, assumindo simetria de fluxo para o restante do molde, como mostrado na Figura 3c.

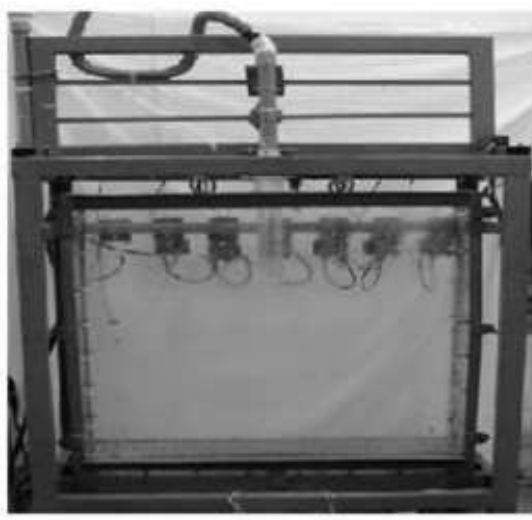

a

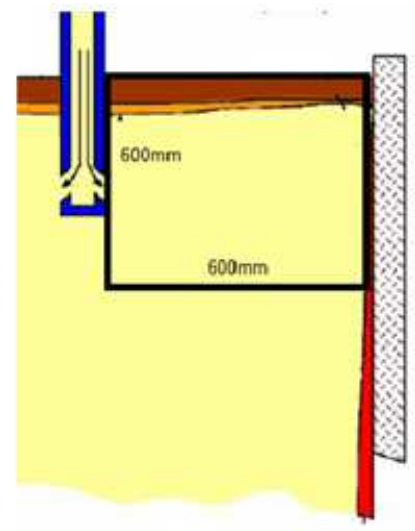

b

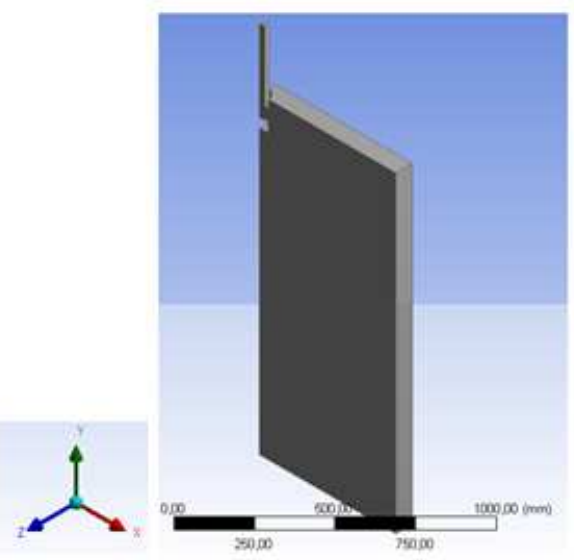

C

Figura 3. a) Modelo físico do molde de lingotamento contínuo (DEMET/EM/UFOP); b) Região de captura de imagens através da técnica PIV para análise de fluxo no molde; c) Geometria do molde de lingotamento contínuo para os testes em modelagem matemática no Ansys Fluent.

\section{RESULTADOS}

Utilizou-se uma válvula submersa padrão com o bocal de saída quadrado e angulação de $15^{\circ}$ negativos. As profundidades de imersão da SEN foram 90 e $110 \mathrm{~mm}$; enquanto que a vazão do fluido variou entre 155 e 195 lpm. Água/óleo de silicone e solução de $\mathrm{ZnCl}_{2} /$ óleo de silicone foram empregados para emulação da escória e do aço. Por meio da técnica PIV obteve-se mapas vetoriais, com curvas de velocidade e energia cinética de turbulência a $1 \mathrm{~cm}$ da interface metal/escória. Foram conduzidas simulações matemáticas, via Ansys Fluent, para as mesmas condições operacionais. A Figura 4 exibe as imagens do molde de lingotamento contínuo e mapas vetoriais correspondente à vazão de $155 \mathrm{lpm}$ e com imersões da SEN de $110 \mathrm{~mm}$ e $90 \mathrm{~mm}$, evidenciando a região do menisco investigada, especificamente na reta traçada no topo. Comparando as duas imersões da SEN, observa-se um maior afinamento da camada de escória em menor imersão da SEN. 

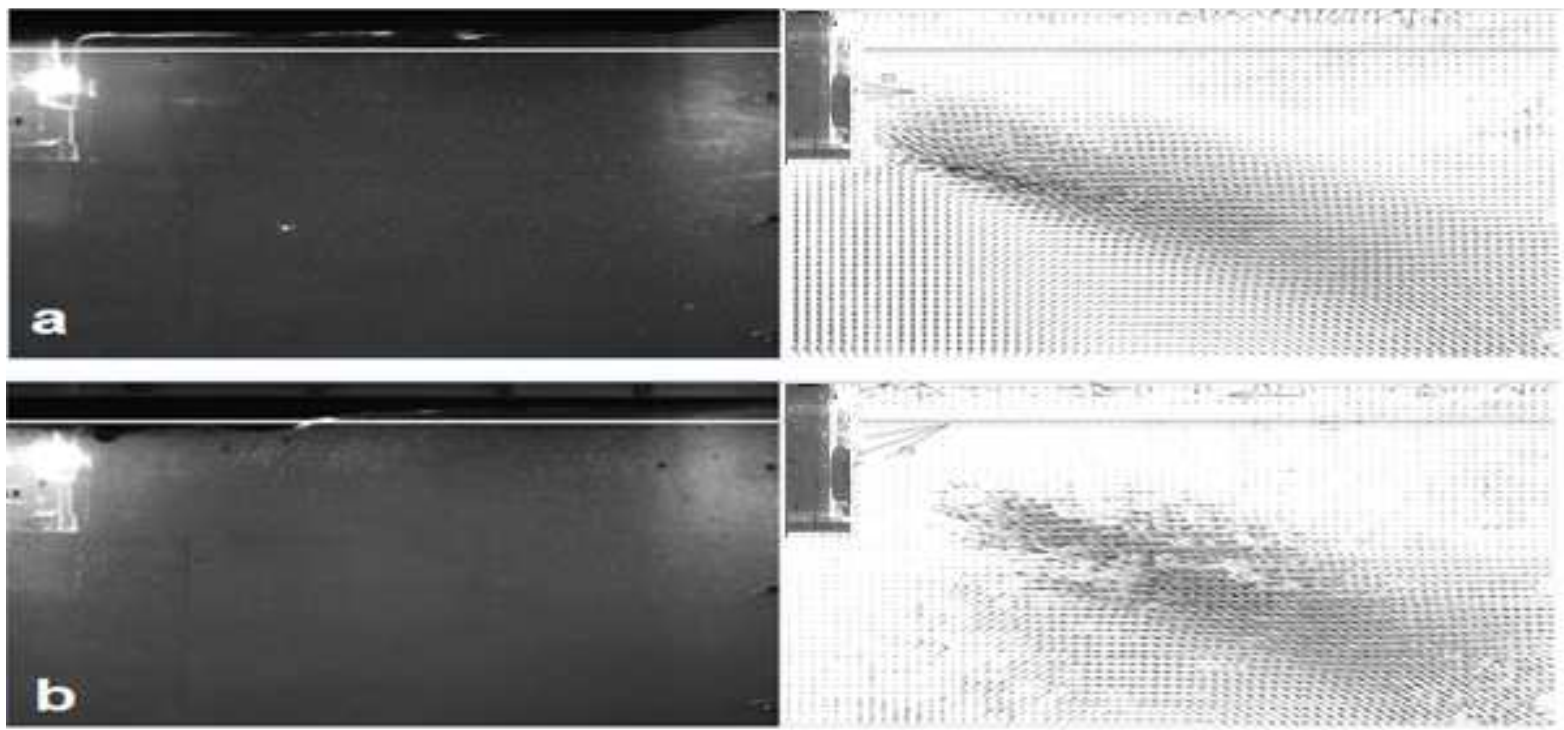

Figura 4. Imagens do molde e mapa vetorial em modelagem física para vazão de $155 \mathrm{lpm}$ de fluido com angulação de 15 da SEN: a) $110 \mathrm{~mm}$ de imersão; b) $90 \mathrm{~mm}$ de imersão.

A Figura 5 retrata a simulação matemática para as mesmas condições de lingotamento, no modelo físico, mostrado na Figura 4. Menores profundidades de imersão da SEN incorrem em maior arraste de escória do molde.
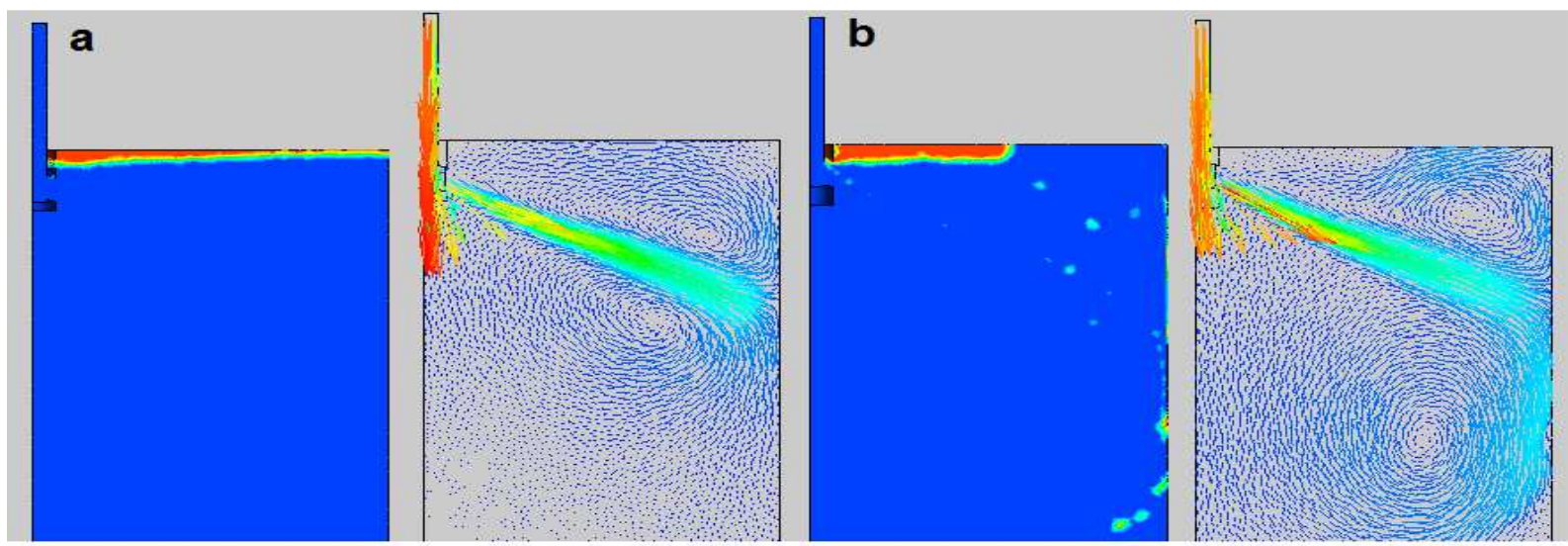

Figura 5. Resultados das condições do menisco e mapa vetorial no Ansys Fluent para vazão de 155lpm e angulação de $15^{\circ}$ da SEN: a) $110 \mathrm{~mm}$ de imersão; b) $90 \mathrm{~mm}$ de imersão.

Os perfis de velocidade e energia cinética de turbulência versus distância a partir da SEN para as duas profundidades de imersões da SEN e vazão de $155 \mathrm{lpm}$ estão exibidos na Figura 6 . Nota-se a ocorrência de altas velocidades junto à face estreita comparativamente com o restante do molde. Para a profundidade de imersão da SEN de $110 \mathrm{~mm}$, os valores de energia cinética de turbulência são próximos para duas modelagens. Enquanto, que para a profundidade de imersão da SEN de $90 \mathrm{~mm}$, prevê-se maiores valores de turbulência via modelagem matemática, principalmente próximo à face estreita. Tanto a velocidade como a energia cinética de turbulência no menisco são maiores quando de menores profundidades de imersão da SEN. 

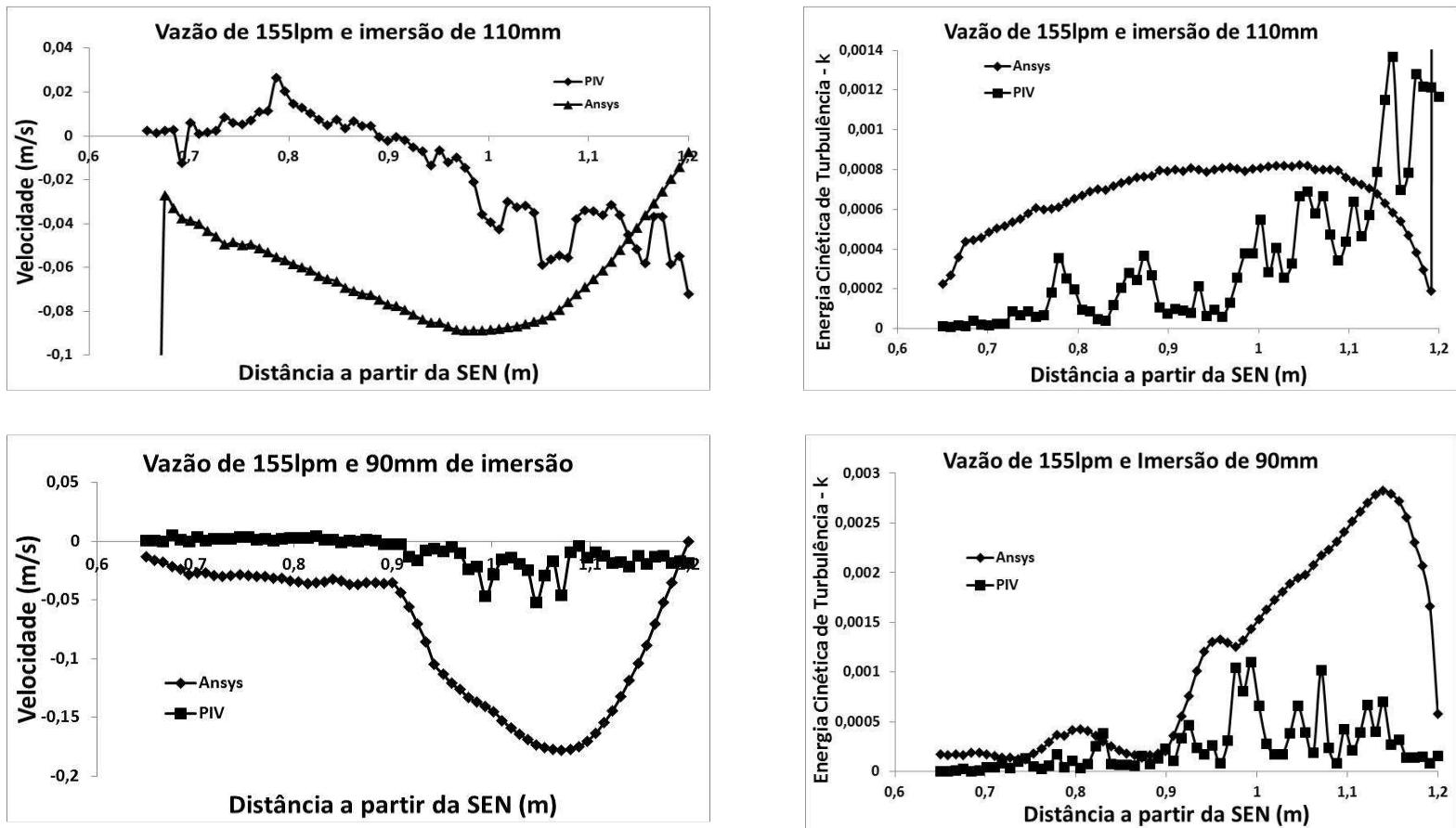

Figura 6. Gráficos de Velocidade e Energia Cinética de Turbulência para vazão de 155lpm nas imersões de $110 \mathrm{~mm}$ e $90 \mathrm{~mm}$ nos estudos físico e matemático.

Para vazão de $175 \mathrm{lpm}$, Figura 7 , as imagens e mapas vetoriais confirmam que menores profundidades de imersão da SEN incorrem em maior deslocamento da camada de escória, o que pode estimular o entranhamento de escória do molde na poça de aço.
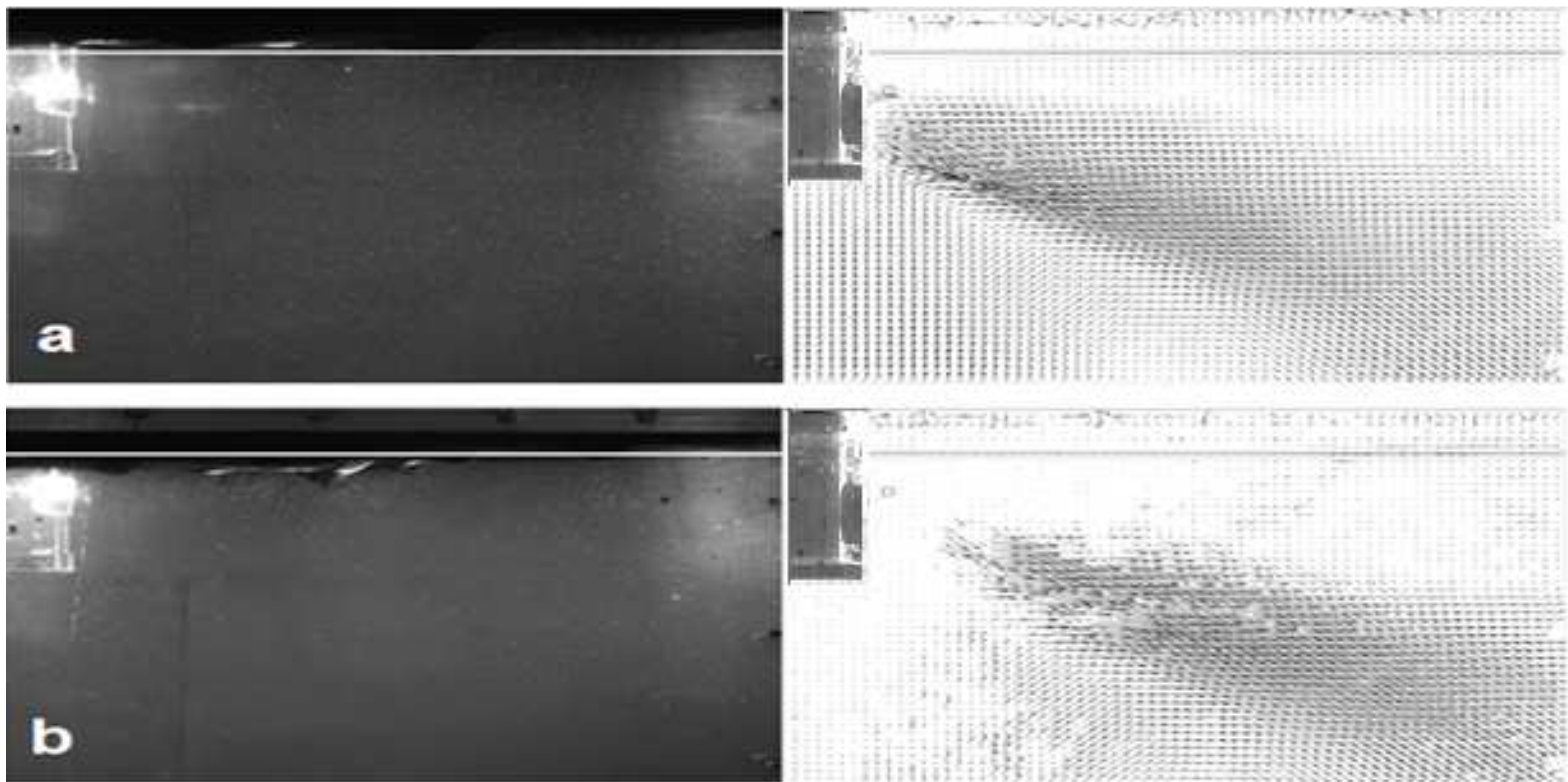

Figura 7.Imagens do molde e mapa vetorial em modelagem física para vazão de $175 \mathrm{lpm}$ de fluido com angulação de 15ำ da SEN: a) $110 \mathrm{~mm}$ de imersão; b) $90 \mathrm{~mm}$ de imersão.

A Figura 8 mostra resultados de simulação matemática em condições idênticas aos da modelagem física da Figura 7. Estes prevêm arraste expressivo de escória para menores profundidades de imersão da SEN, o que não foi observado na modelagem física. 

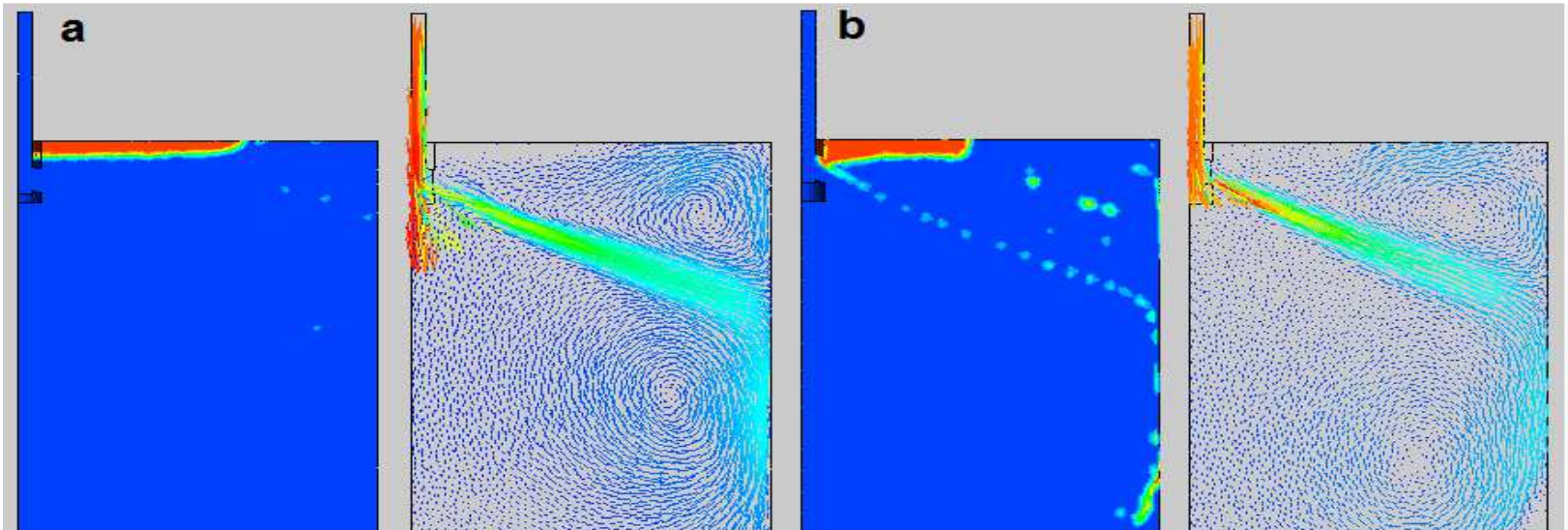

Figura 8. Resultados das condições do menisco e mapa vetorial no Ansys Fluent para vazão de 175lpm e angulação de 15ํำ da SEN: a) $110 \mathrm{~mm}$ de imersão; b) $90 \mathrm{~mm}$ de imersão.

A Figura 9 mostra gráficos de velocidade e energia cinética de turbulência para as duas imersões da SEN na vazão de 175lpm, em ambas modelagens. Observa-se, para as duas imersões e ambos os modelamentos, altos valores de velocidade próximo à face estreita se comparado ao restante da região do molde. Estas são maiores nas simulações matemáticas e prevêm então um maior deslocamento da camada de escória. As curvas de energia cinética de turbulência mostram a mesma tendência das curvas de velocidade. As duas imersões da SEN apresentam valores próximos de velocidade, porém maiores valores de energia cinética de turbulência são observados em menores imersões da SEN.
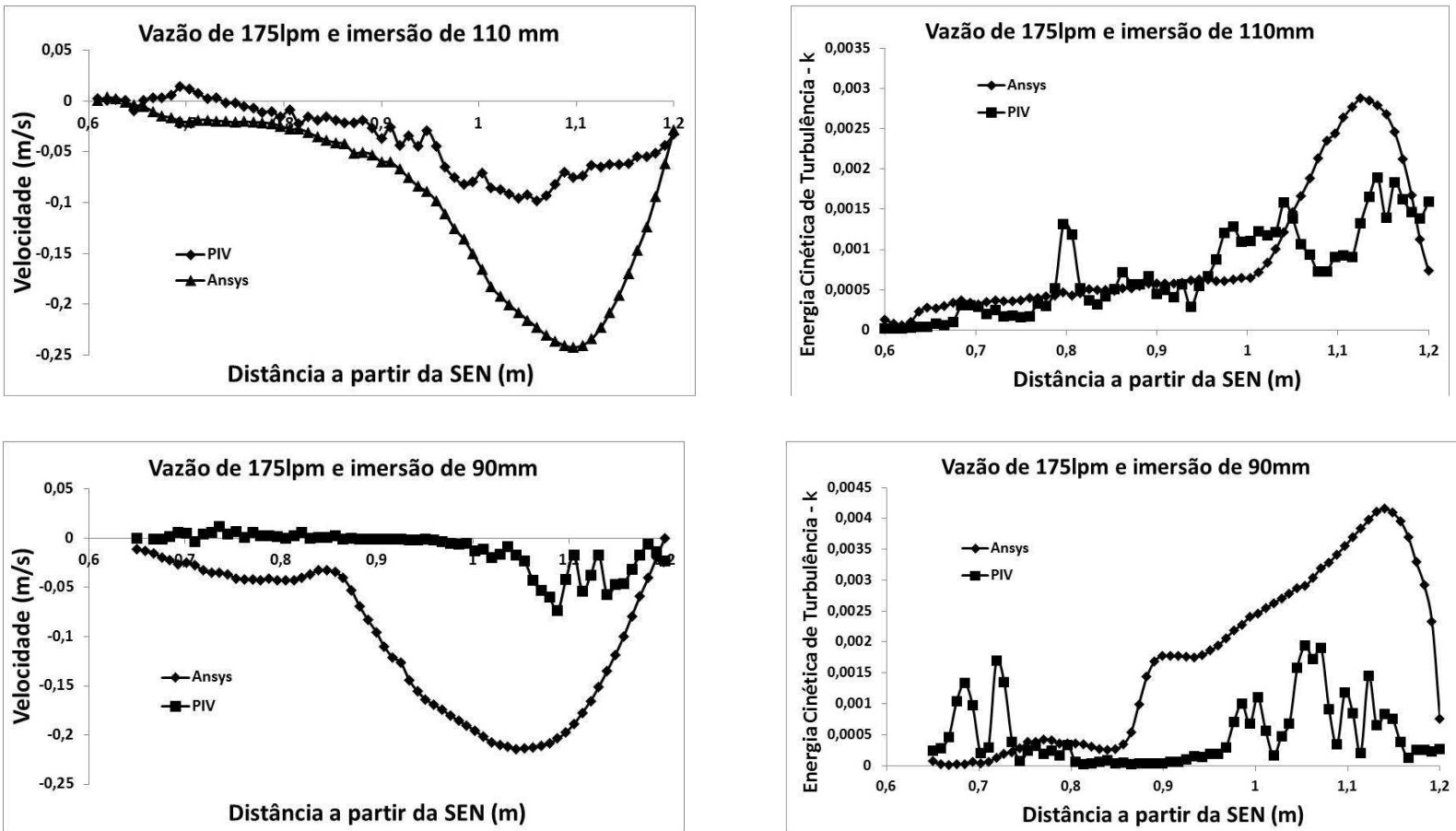

Figura 9. Gráficos de Velocidade e Energia Cinética de Turbulência para vazão de 175lpm nas imersões de $110 \mathrm{~mm}$ e $90 \mathrm{~mm}$ nos estudos físico e matemático.

O mesmo estudo foi realizado para a vazão de 195lpm. A Figura 10 mostra que assim como nas menores vazões, em menores imersões da SEN, há um maior deslocamento da camada de escória para esta condição, havendo uma maior deformação próximo à face estreita e arraste de escória ao longo dos experimentos. 

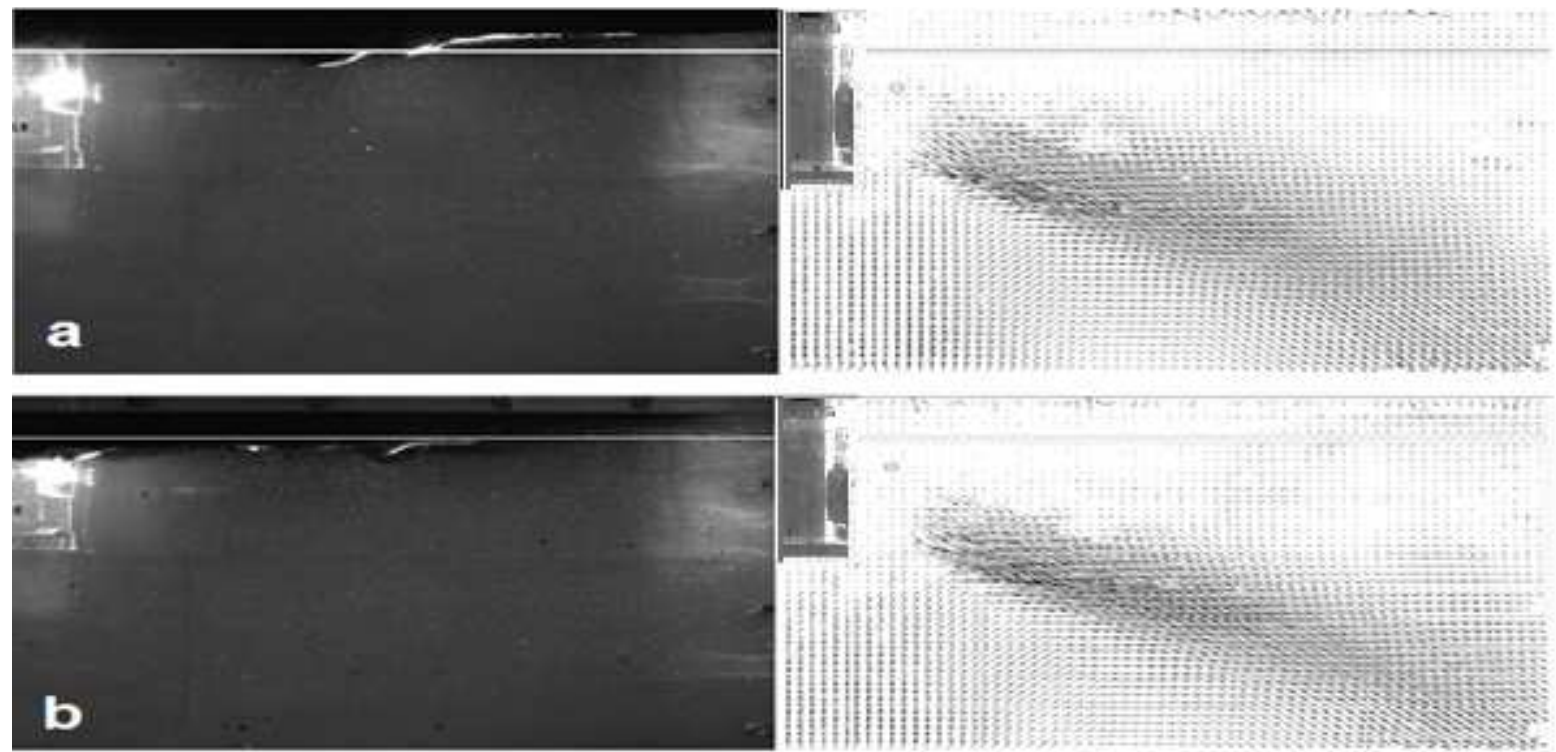

Figura 10.Imagens do molde e mapa vetorial em modelagem física para vazão de 195lpm de fluido com angulação de $15^{\circ}$ da SEN: a) $110 \mathrm{~mm}$ de imersão; b) $90 \mathrm{~mm}$ de imersão.

A Figura 11 mostra imagens da simulação matemática nas condições idênticas aos da Figura 10. Não existe, como antes, equivalência perfeita entre as modelagens física e matemática. O entranhamento, eventual, de escória foi observado na modelagem física; entretanto a simulação matemática prevê este fenômeno de forma intensa.

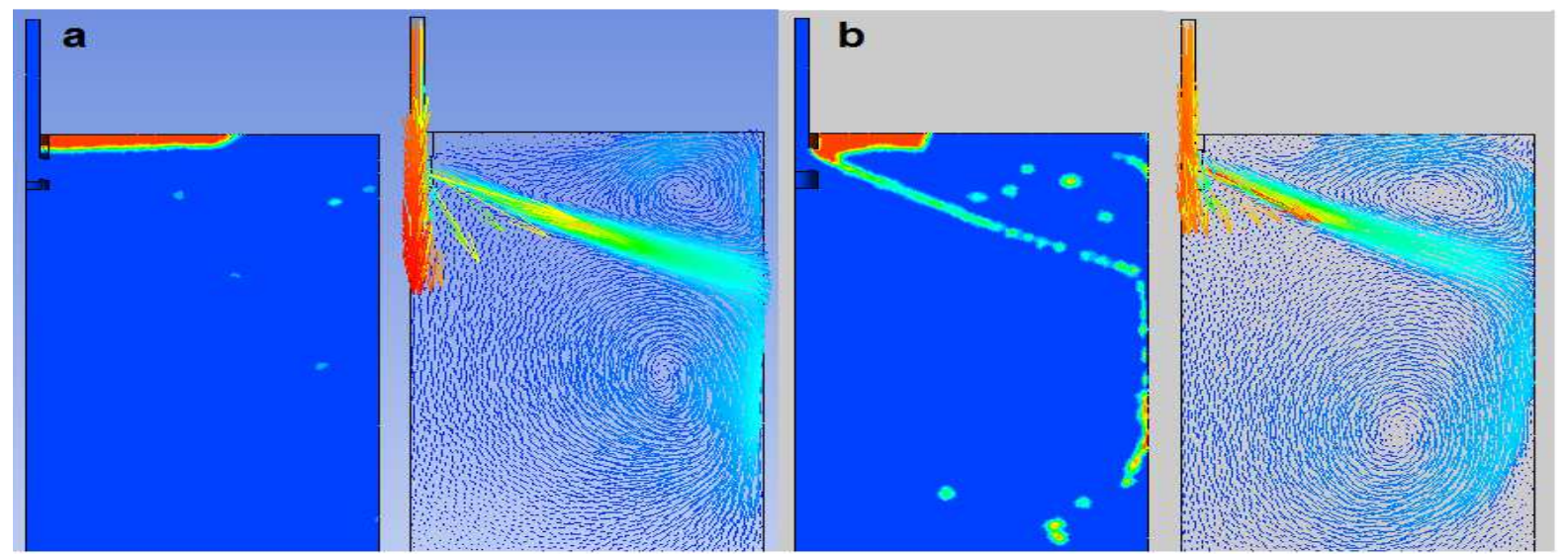

Figura 11. Resultados das condições do menisco e mapa vetorial no Ansys Fluent para vazão de 195lpm e angulação de 15ำ da SEN: a) 110mm de imersão; b) 90mm de imersão.

A Figura 12 mostra gráficos de velocidade e energia cinética de turbulência para a vazão de 195/pm. Observa-se que para as duas imersões e nos dois modelamentos há altos valores de velocidade e turbulência próximo à face estreita se comparado ao restante da região do molde. Nota-se, como esperado, maior turbulência no menisco para a menor imersão da SEN.

Discrepâncias apontadas neste trabalho entre os modelos físico e matemático se devem também ao fato que o PIV reflete uma média de 10s de operação em regime (pseudo) permanente, enquanto que os da simulaçaõ matemática retratam o sistema após 10 s do início de um processo no qual, inicialmente, os fluidos estão em repouso. 

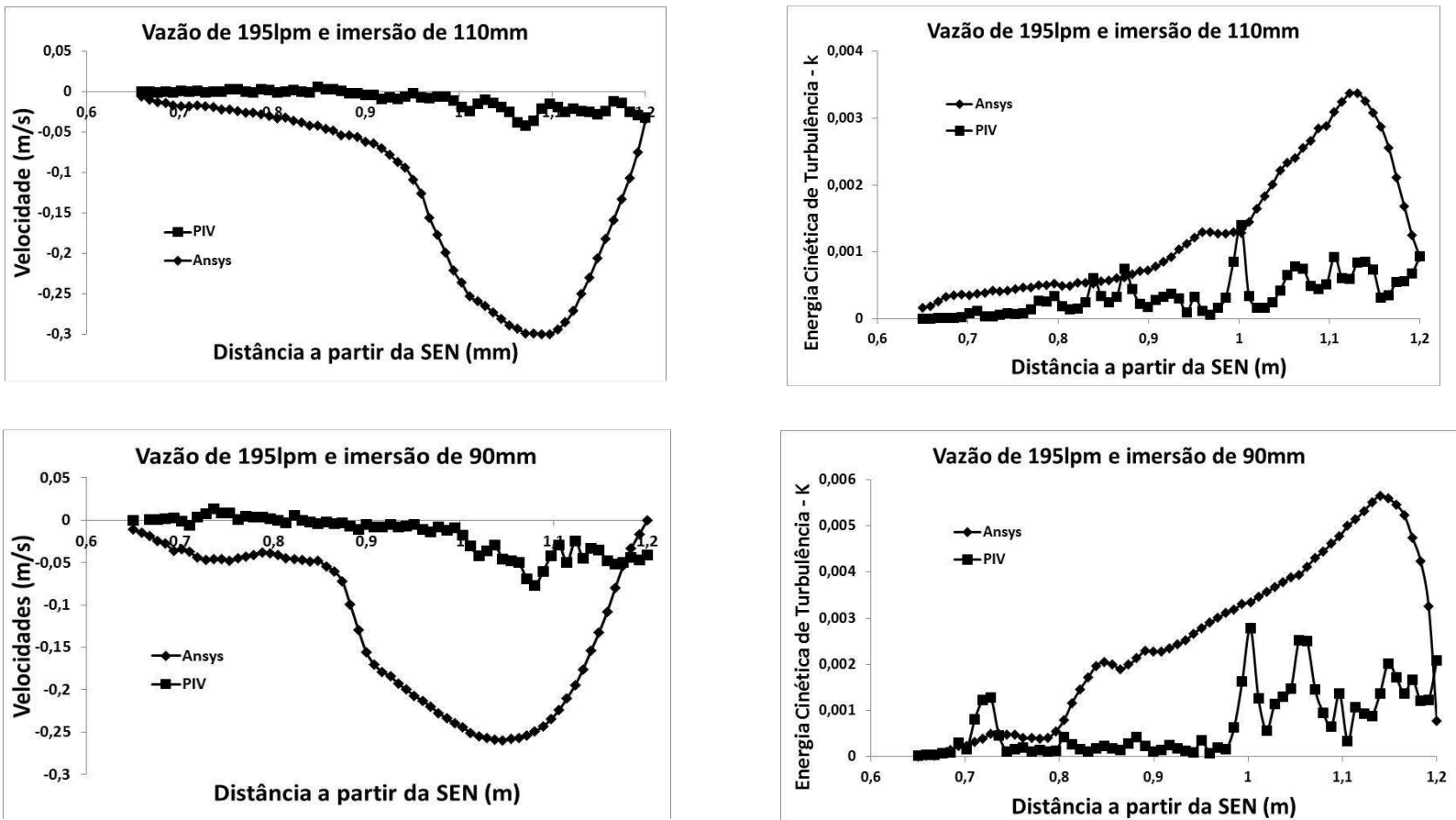

Figura 12. Gráficos de Velocidade e Energia Cinética de Turbulência para vazão de 195lpm nas imersões de $110 \mathrm{~mm}$ e $90 \mathrm{~mm}$ nos estudos físico e matemático.

Fluidos diferentes foram utilizados para simular aço/escória. A Figura 13 mostra imagens para a vazão de 155lpm, nas duas imersões de $90 \mathrm{~mm}$ e $110 \mathrm{~mm}$ da SEN. Observa-se uma menor movimentação da camada da escória no sistema solução de $\mathrm{ZnCl}_{2}$, pois menores turbulências são observadas na região do menisco.

a

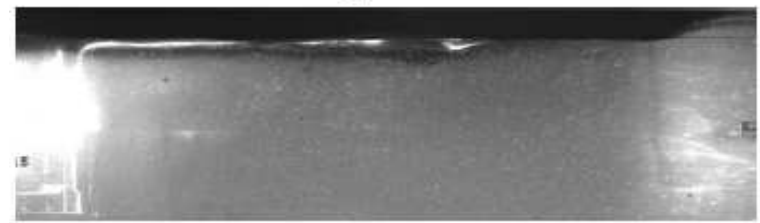

C b

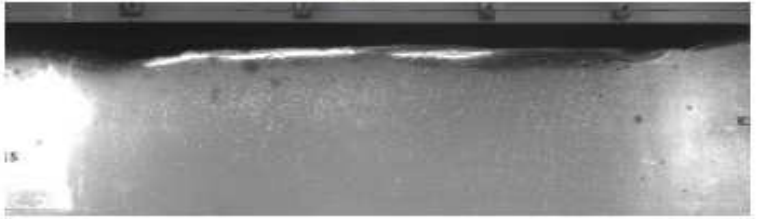

d
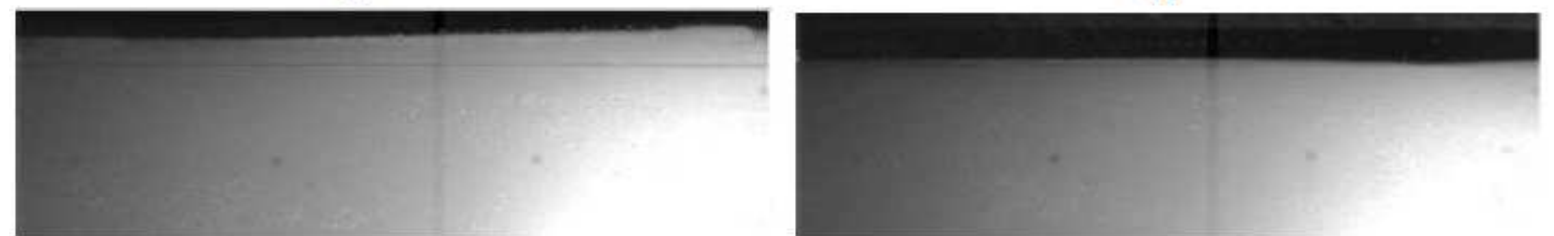

Figura 13 - Imagens da interface. a) água/óleo $V=155 \mathrm{lpm}, \mathrm{Im}=110 \mathrm{~mm}$; b) água/óleo $\mathrm{V}=155 \mathrm{Ipm}$, Im=90mm; c) solução $\mathrm{ZnCl}_{2} \mathrm{~V}=155 \mathrm{lpm}$, Im=110mm; d) solução $\mathrm{ZnCl}_{2} \mathrm{~V}=155 \mathrm{lpm}$, Im=90mm.

A diferença principal entre estes dois sistemas, ver tabela 1 e tabela 2 , se deve à diferença de densidade entre os fluidos: $50 \mathrm{~kg} / \mathrm{m}^{3}$ nas simulações com água e $350 \mathrm{~kg} / \mathrm{m}^{3}$ nas simulações com $\mathrm{ZnCl}_{2}$. Maiores diferenças de densidade tendem a promover maior estabilidade da interface, como será destacado a seguir. A Figura 14 apresenta o caso da maior vazão, 195lpm, para o qual se notou entranhamento da camada de escória no sistema água e óleo. Mas não no sistema óleo - solução de $\mathrm{ZnCl}_{2}$. 
a

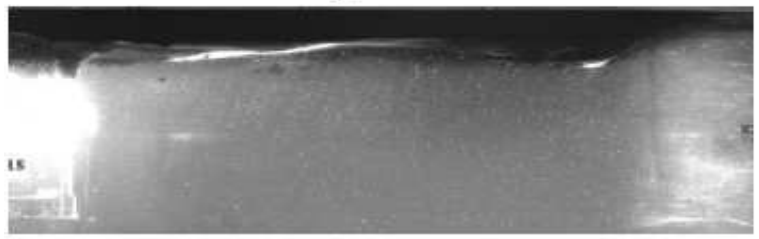

c

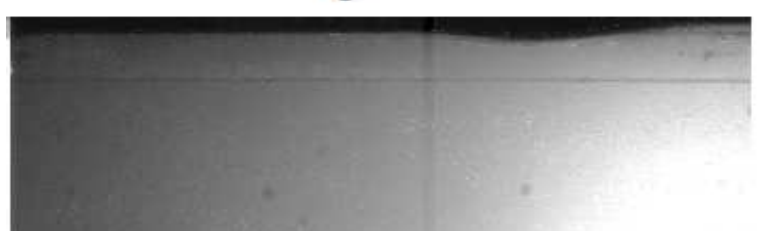

b

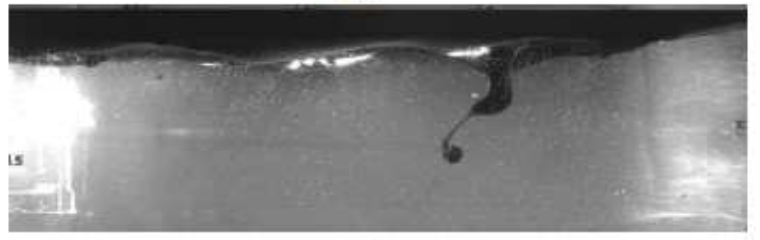

d

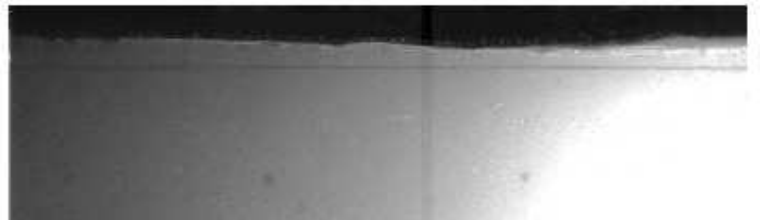

Figura 14 - Imagens da interface. a) água/óleo V=195lpm, Im=110mm; b) água/óleo V=195lpm, Im=90mm; c) solução $\mathrm{ZnCl}_{2} \mathrm{~V}=195 \mathrm{lpm}$, Im=110mm; d) solução $\mathrm{ZnCl}_{2} \mathrm{~V}=195 \mathrm{lpm}$, Im=90mm.

As imagens indicam nítida diferença da flutuação na interface metal/escória para os dois sistemas analisados. A ondulação causada na camada de óleo no sistema água/óleo mesmo para as menores vazões e o arraste de escória, quando da vazão de $195 \mathrm{lpm}$ e imersão de $90 \mathrm{~mm}$, mostram as condições críticas deste sistema com relação ao de solução de $\mathrm{ZnCl}_{2} /$ óleo. Quanto maior a diferença de densidade entre os fluidos que simulam aço/escória, menores são as flutuações na região do menisco. De acordo com Cramb [5], a velocidade mínima de entranhamento de escória é correlacionada com propriedades físicas do aço e escória, tal que:

$$
V_{\text {ent }}=3,065 * \frac{\gamma^{0,292} * g^{0,115} * \Delta \rho^{0,215} * \mu_{\text {óleo }}{ }^{0,231}}{\rho_{\text {óleo }}{ }^{0,694} * \Delta d^{0,365} * \mu_{\text {água }}, 043}
$$

onde: $\gamma=$ tensão interfacial metal/escória $; \rho=$ massa específica $; \Delta d=$ espessura da camada de escória ; $\mu=$ viscosidade. Por meio desta correlação matemática, podese prever uma relação entre as velocidades críticas entre os dois sistemas. Utilizando os valores de propriedades de fluidos apresentados nas Tabelas 1 e 2, chega-se ao valor de $\mathrm{V}_{\text {ent(água/óleo) }}=0,0473$ e $\mathrm{V}_{\text {ent(Zncl2/óleo) }}=0,0625$. Portanto, a razão entre esses valores de velocidade mostra que a velocidade de lingotamento do sistema água/óleo de silicone é $32 \%$ maior que o sistema $\mathrm{ZnCl}_{2} /$ óleo de silicone.

De acordo com Kasai (apud Suzuki [6], 2001) o destacamento de uma gota de escoria ocorre quando as forças de tensão interfacial e de empuxo deixam de ser capazes de garantir a estabilidade da interface. Daí a velocidade crítica deve ser função do grupo envolvendo estas forças,

$V \propto\left(\frac{\sqrt{\Delta \rho g \gamma}}{\rho_{\text {óleo }}}\right)^{1 / 2}$

Esta análise dá um peso ainda maior ao empuxo e sugere que no sistema óleo solução de $\mathrm{ZnCl}_{2}$ as velocidades críticas são maiores, em cerca de $60 \%$. Claramente, como mostram as simulações, o fenômeno de emulsificação é amplificado nos experimentos envolvendo água e óleo. Isto pode explicar a diferença no comportamento dos fluidos na região do menisco.

Tabela 1 - Propriedades específicas de materiais utilizados nas simulações em laboratório.

\begin{tabular}{|c|c|c|}
\hline MATERIAL & DENSIDADE $\left(\mathrm{Kg} / \mathrm{m}^{3}\right)$ & VISCOSIDADE \\
\hline Água & 1000 & $1,0 \mathrm{cSt} ; 0,001 \mathrm{~Pa} . \mathrm{s}$ \\
\hline Solução de água com $\mathrm{ZnCl}_{2}$ & 1300 & $2,33 \mathrm{cSt} ; 0,00303 \mathrm{~Pa} . \mathbf{s}$ \\
\hline Óleo de Silicone & 950 & $500 \mathrm{cSt} ; 0,526 \mathrm{~Pa} . \mathrm{s}$ \\
\hline Querosene & 750 & $3,0 \mathrm{cSt} ; 0,00225 \mathrm{~Pa} . \mathrm{s}$ \\
\hline
\end{tabular}


Tabela 2 - Tensão Interfacial dos sistemas utilizados nas simulações a $20^{\circ} \mathrm{C}$.

Água/Óleo de Silicone TENSÃO INTERFACIAL (N/m)

$\mathrm{ZnCl}_{2} /$ Óleo de Silicone

0,0357

0,0261

\section{CONCLUSÃo}

Os resultados obtidos permitem inferir que:

- Maiores vazões de lingotamento e menores profundidades de imersão incorrem em maiores deformações da camada de escória;

- A modelagem matemática prevê maiores deformações da camada de escória e maiores valores de velocidade e energia cinética de turbulência comparativamente com a modelagem física, entretanto, a tendência de comportamento foi a mesma. As discrepâncias são, entretanto, também devidas ao modo de amostragem.

- Maiores diferenças de densidade e maiores valores de tensão interfacial efetivamente contribuem para uma maior estabilidade da interface.

\section{Agradecimentos}

À FAPEMIG e ao CNPq pelo apoio financeiro à esta pesquisa.

\section{REFERÊNCIAS}

1 JABRI, K - Etude et amélioration des performances et de la robustesse des lois de commande de procédés sidérurgiques Application à la régulation de niveau en coulée continue, Thése de Doctorat, Univesitè Paris Sud 11, 2010.

2 THOMAS, B.G.; HIBBELER, L.C. Investigation of Mold Flux Entrainment in CC Mold Due to Shear Layer Instability, Assoc. Iron Steel Technology, Warrendale, PA, CCC Report 201001, Aug. 2010.

3 ZARE, M.H.; MEYSAMI, A.H.; MAHMOUD, SH.; HAJISAFARI, M.; ATABAKI, M.M. Simulation of flow field and steel/slag interface in the mold region of a thin slab steel continuous caster with tetra-furcated nozzle, Journal of Manufacturing Processes, 2013.

4 BARRAL C. E. P "Efeito de parâmetros geométricos e operacionais sobre o comportamento do pó fluxante no molde de lingotamento contínuo de placas", Dissertação de Mestrado, Universidade Federal de Minas Gerais, Brasil, 2006.

5 HARMAN J. M., CRAMB A. W. A Study of the Effect of Fluid Physical Properties upon Droplet Emulsification, Steelmaking Conference Proceedings, 1996, p. 773-784.

6 Suzuki M., Suzuki M., Nakada M., Perspectives of Research on High-speed Conventional Slab Continuous Casting of Carbon Steels, ISIJ International, vol 41(2001), no 7, pp 670682 